MATHEMATICS OF COMPUTATION

Volume 71, Number 239, Pages 1337-1338

S $0025-5718(02) 01487-4$

Article electronically published on April 1, 2002

\title{
CORRIGENDA TO
}

\section{"NEW PRIMITIVE $t$-NOMIALS $(t=3,5)$ OVER $G F(2)$ WHOSE DEGREE IS A MERSENNE EXPONENT," AND SOME NEW PRIMITIVE PENTANOMIALS}

\author{
TOSHIHIRO KUMADA, HANNES LEEB, YOSHIHARU KURITA, \\ AND MAKOTO MATSUMOTO
}

\begin{abstract}
We report an error in our previous paper [2], where we announced that we listed all the primitive trinomials over $G F(2)$ of degree 859433 , but there is a bug in the sieve. We missed the primitive trinomial $X^{859433}+$ $X^{170340}+1$ and its reciprocal, as pointed out by Richard Brent et al. We also report some new primitive pentanomials.
\end{abstract}

\section{Corrigendum}

In 2, Table 1], we claimed that all primitive trinomials with degree 859433 (32nd Mersenne exponent) over $G F(2)$ are $X^{859433}+X^{288477}+1$ and its reciprocal, but there was a bug in a code for the sieve. Richard Brent et al. 11 pointed out that there are two more primitive trinomials of this degree: $X^{859433}+X^{170340}+1$ and its reciprocal, through their complete search for the primitive trinomials of degrees 756839, 859433, and 3021377 [1]. (The primitivity of the above trinomial was confirmed by our corrected code, too.) Their current search is shown on the website

http://web.comlab.ox.ac.uk/oucl/work/richard.brent/trinom.html.

\section{NEW PRIMITIVE PENTANOMIALS}

Here we report that the following primitive pentanomials (see Table 1 on the next page) have been found by the method described in [2].

\section{ACKNOWLEDGMENTS}

We would like to thank Professor R. Brent and his colleagues for pointing out our error.

Received by the editor May 10, 2001.

2000 Mathematics Subject Classification. Primary 11-04, 11T06, 12-04, 12 E05.

Key words and phrases. Irreducible polynomials, primitive polynomials, finite field, Mersenne exponent. 
TABLE 1. Some of $p, q_{1}, q_{2}, q_{3}$ for which $X^{p}+X^{q_{1}}+X^{q_{2}}+X^{q_{3}}+1$ is primitive over $G F(2)$

\begin{tabular}{|rrrr|}
\hline$p$ & $q_{1}$ & $q_{2}$ & $q_{3}$ \\
\hline \hline 44497 & 28473 & 25357 & 6183 \\
44497 & 28927 & 18413 & 7668 \\
44497 & 33021 & 19223 & 12151 \\
44497 & 34275 & 26980 & 9923 \\
44497 & 35043 & 27313 & 6311 \\
44497 & 38802 & 23900 & 6536 \\
\hline 86243 & 61388 & 32606 & 26237 \\
86243 & 61995 & 49334 & 25248 \\
86243 & 65723 & 41510 & 30407 \\
86243 & 67935 & 50330 & 22621 \\
86243 & 68677 & 42129 & 11704 \\
86243 & 69017 & 46561 & 26682 \\
86243 & 69098 & 41740 & 13977 \\
86243 & 69453 & 41544 & 12701 \\
86243 & 69615 & 51770 & 17232 \\
\hline
\end{tabular}

\begin{tabular}{|rrrr|}
\hline$p$ & $q_{1}$ & $q_{2}$ & $q_{3}$ \\
\hline \hline 110503 & 71270 & 66923 & 19978 \\
110503 & 75061 & 42595 & 36334 \\
110503 & 77029 & 67563 & 40579 \\
110503 & 78339 & 65279 & 14642 \\
110503 & 78832 & 42854 & 27560 \\
110503 & 80053 & 48219 & 27930 \\
110503 & 80069 & 42319 & 32108 \\
110503 & 81999 & 66969 & 26952 \\
110503 & 88763 & 63837 & 31613 \\
110503 & 89629 & 48590 & 20837 \\
110503 & 89758 & 57438 & 39069 \\
110503 & 92048 & 53327 & 15882 \\
110503 & 92797 & 61896 & 21698 \\
110503 & 93253 & 61728 & 21110 \\
110503 & 93750 & 46605 & 29808 \\
110503 & 95508 & 64105 & 37825 \\
\hline
\end{tabular}

\section{REFERENCES}

1. R. P. Brent, S. Larvala and P. Zimmermann, A fast algorithm for testing irreducibility of trinomials mod 2 (preliminary report), Report PRG TR-13-00, 30 December 2000. Available from http://web.comlab.ox.ac.uk/oucl/work/richard.brent/pub/pub199.html.

2. T. Kumada, H. Leeb, Y. Kurita and M. Matsumoto, New primitive $t$-nomials $(t=3,5)$ over $G F(2)$ whose degree is a Mersenne exponent, Math. Comp. 69 (2000), no. 230, 811-814. MR 2000i:11183

Daiwa Institute of Research Ltd. 15-6 Fuyuki, Koto-ku, Tokyo 135-8460, Japan

E-mail address: t.kumada@dir.co.jp

Institute of Statistics, University of Vienna, Universitaetsstr. 5, 1010 Vienna, Austria

E-mail address: Hannes.Leeb@univie.ac.at

Nippon Electric Control Equipment Industries Association, 2-1-17 Hamamatsu-Cho, MinATO-KU, TOKYO 105-0013 JAPAN

E-mail address: ykurit@attglobal.net

Division of Mathematics, Integrated Human Studies, Kyoto University, Kyoto 6068501 JAPAN

E-mail address: matumoto@math.h.kyoto-u.ac.jp 